will not strike it at the very beginning of the act of impulse, but just at the instant when the fall force of the apex beat has been communicated to the wire. It is essier to use this appliance along with the binaural stethoscope, as the operator can thus have the use of his eyes in adjusting the mechanism. After fixing the disc and wire in situ it is advisable to apply the stethoscope, and listen carefully for a few moments to the heart scunds before bringing the wire in contact with the wood. In this way the intensity and quality of the sounds or murmurs, and the length of the pauses, etc., can be examined and appreciated when, the sharp rap of the wire on the wood being induced, the rhythm will be demonstrated.

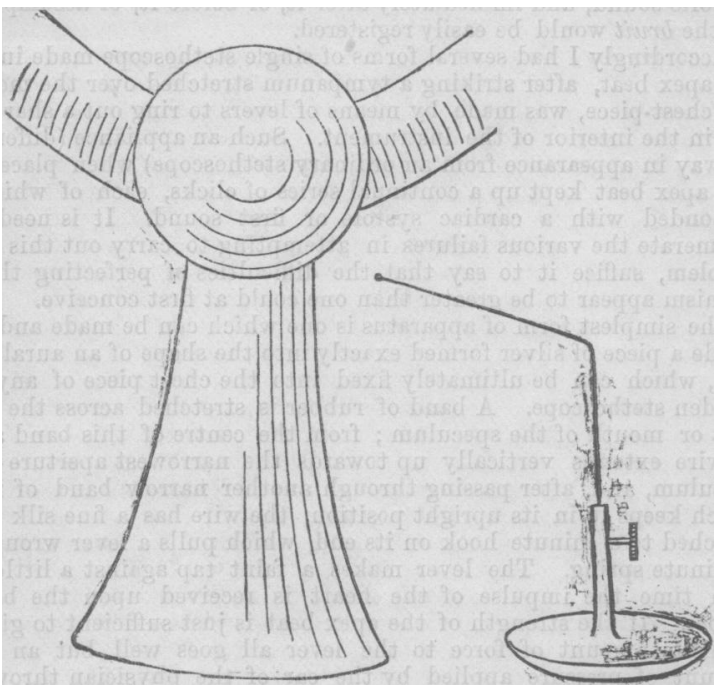

This little instrument, which for convenience might be termed a systolqmeter, may be modified in various ways; thus I have replaced the metallic disc with a minute cup-shaped piece of india-rubber, such as is used for suspending small articles from the polished surfaces of window panes or mirrors. The rubber being pressed firmly against the moistened skin will adhere like the leather sucker known to every schoolboy, and will remain in situ sometimes sufficiently long to allow of examination. It is needless to say that the systolometer can only be used when a distinct impulse is present.

$\Delta$ very satisfactory form of systolometer can be extemporised by attaching a section of a wine or lemonade cork over the apex region, as referred to by Dr. Dickinson in his recent paper, and after securing it in position with strips of adhesive plaster, a piece of bent lemonade wire having been inserted into the cork can be used as a lever to strike the bell of the stethoscope, and mark the moment at which the natura first sound of the heart should be heard.

In a future contribution I hope to be able to give the results of this simple mechanism, applied to the investigation of some doubtful problems concerning the working of the healthy heart and the mechanism of the presystolic murmur. That the principle is correct upon which its usefulness depends there cannot be any doubt, and its application is now suggested with the hope that ir the hands of abler observers its form will be improved and -perfected, so that to the student especially its use may be an advantage.

\section{TWO CASES OF CHOLECYSTOTOMY.}

\section{BY J. KNOWSLEY THORNTON, M.B., C.M.,} Burgeon to the Samaritan Free Hospital.

THE need for the performance of cholecystotomy may arise from impaction of a gall stone in the cystic duct, or from closure of this dact in some other way ; in such a case we have to deal with a distended gall bladder, its flaid contents not necessarily, or, indeod, commonly, being bile, but a serous, sero-purulent, muco-puralent or purulent flaid. In such a case there will be the distended gall bladder palpable in the abdomen to help our diagnosis, and there will be the fear that it may rupture into the peritoneum to arge us on to attempt operative relief. Jaundice is not present in these cases.
The operative procedure is easy ; the distended gall bladder allows plenty of room for the extraction of the stone or stones, and at the close of the operation its stretched walls can be readily sutured into the abdominal wound, the peritoneal cavity effectively closed, and the unhealthy gall bladder drained exterually as long as necessary. The published records of such operations show that they are attended with but trifling risk in the hands of experienced operators.

The two cases I am about to describe differ in several particulars from the above, the differences being due to the important fact that the stones had passed into the common duct before impaction took place.

Both patients were deeply jaundiced, both were in very broken general health, and both had markedly enlarged livers, but in neither could the gall bladder be distinctly defined.

I will now proceed to describe the cases in some detail, and will conclude with remarks upon the method to be fullowed in operating when the stone is impacted in the cystic duct, and when it has passed into the common duct, leaving, as in the following cases, a much contracted gall bladder behind it.

Casm I. - In February of this year (1887) I was requested by my friend Dr. George Johnson to see with him at the Euston Hotel a lady who gave us the following history.

H. B., aged 45, married when 28, and mother of seven children, the youngest being $4 \frac{1}{2}$ years old. Very healthy both before and after marriage, but during the last ten years has suffered from bronchial asthma in the winter. Sight failed a good deal in 1885, and being told by an oculist that this was due to general debility, she consulted Dr. Johnson early in 1886. He found her suffering from indigestion and congestion of the liver. Under his care she greatly improved in health, but about a fortnight after her return home she was suddenly seized one evening with violent pain over the liver and vomiting, which continued all night, and in the morning she was jaundiced. She was very ill, and Dr. Foster, of Birmingham, saw her in consultation. This illness lasted nine weeks, and she was then sent to Torquay, and at the end of three weeks had another attack of pain, followed by jaundice, which Dr. Huxley attributed to cold. She returned home in December, and almost immediately began to suffer with repeated attacks of pain over the liver and in the back, accompanied by taintness and such prostration that she was soon quite confined to her room. Rapid emaciation now set in, and she came up to London again.

When I saw her with Dr. Johnson she was deeply jaundiced, anæmic, and emaciated, with that peculiar loose skin which denotes recent and rapid emaciation. She complained of almost constant pais deep down in the region of the gall bladder and in the back. Carefal percussion showed a much enlarged liver, but no special projection of the dull area in the region of the gall bladder. On deep palpation which caused her great pain, I thought I could distinctly feel a hard angular body. It seemed clear, both to Dr. Johnson and myself, that she had a stone impacted in the common duct, and that her state of health was such as to cause grave anxiety. The fear that ulceration and perforation might occur at any time influenced us in advising the early removal of the stone.

The risks of expectant treatment and of operative treatment having been duly explained to herself and her husband, they decided upon operation, and on February 8th, in the presence of Dr. Johnson, Mr Malcolm assisting, and Mr. Murray administering chloroform, I exposed the gall bladder by a short vertical incision (about three inches) immediately over its site. The gall bladder was so strophied and sur rounded by adherent omentum and small intestine, that I at first thought it was non-existent; but I speedily found the stone in the situation in which I had believed I felt it before operation. Carefully scratching through the adherent omentum, I at last found the outex surface of the gall bladder, and, aspirating, drew off about a drachm of muco-purulent fluid. I should have said that, before making this puncture, the gall bladder was carefully packed round with carbolised sponges. Finding there was so little fluid, I withdrew the trocar, and, slipping a probe-pointed bistoury through the puncture, enlarged the opening till I could introduce my finger; during this process, fluid kept oozing up in small quantity, the last to be sponged away being glairy bile. The gall bladder was so shrunk that it would only just admit my index finger, and I could feel at its termination the still smaller cystic duct; passing a probe through this duct, I could feel the stone firmly fixed in the common duct; and then began the difficult part of the operation : first, I had carefally to dilate the cystic duct till I could get my finger through it, and feel the exact size and position of the stone, and then I had still further to dilate it, so that I could pass through it, beside my finger, various probes, scoops, forceps, etc. with which to disledge and extract the stone. At last, when I had 
broken it a good deal, and almost despaired as to the removal of the larger fragment, I managed to turn it round, and then, catching its edge, extracted it with these forceps which I use for sponging the pelvis in abdominal section.

This part of the operation occupied over an hour and a half, and the walls of the gall bladder were much torn and bruised by the forceps with which it was necessary to hold up the two sides of the opening into it. Having carefully cleaned out the gall bladder and ducts, with solution of corrosive sublimate 1 to 1,000 , I plugged it with small pieces of sponge soaked in the same solution; and, having sponged out the peritoneum, I attempted to follow the usual course, and suture the edges of the gall bladder into the abdominal incision. This I found to be quite impossible, partly owing to the damaged state of the gall bladder, and partly owing to its atrophied condition. I now determined to perform a careful suture, so as to entirely close the wound in the gall bladder, and leave it free in the peritoneum; even this was accomplished with great difficulty, and I was obliged to utilise to some extent the torn edges of the adherent omentum, in order to get a good hold for my sutures, and thoroughly close the opening. I used a few points of fine interrupted silk suture, and then a fine continuous one over all, this latter one drawing the edges of the torn omentum over the united edges of the wound in the gall bladder, which it also superficially included. On sponging out the peritoveum I found that a certain amount of bloody serum had oozed down into the pelvis, and I determined to make an opening through the abdominal parietes, just above t: $\bullet$ pubes, and put a glass tube in ; I also placed another glass tube in the peritoneal pouch on the right side; the wisdom of the double drainage was abundantly proved by the amount of bloody serum drawn from each during the first few days after the operation. The mouth of each tube was surrounded by dry carbolised gauze, and each had its rubber sheet filled with carbolised sponges, which were changed every twelve hours under the spray, the fluid in the tubes being at the same time sucked out by a piece of red rubber tube attached to a glass syringe.

The case was treated as I treat all my abdominal sections, that is, the patient was kept on her back with the knees raised over a pillow. Twenty drops of laudanum were given in an enema of three ounces of beef tea every six hours, and three ounces of beef tea were also injected every three hours. The temperature slowly mounted till the middle of the day after the operation, when it was $102.2^{\circ}$, with pulse 100 ; pounded ice in a piece of macintosh was then placed on the head, and the temperature quickly fell to $100^{\circ}$, but again mounted to $102^{\circ}$ on the second afterncon, when she had a sharp asthmatic attack. There was no sickness at all. The bloody serum from both tubes was more or less bile-stained, but I do not think this was from the escape of bile from the gall bladder into the peritoneum, but simply because all her tissues were saturated with bile. Both tubes were removed on the sixth day, when the temperature and pulse were normal, and so remained.

During these early days there was a good deal of trouble from the asthmatic breathing and cough, and this, I have no doubt, made the tubes irritate the peritoneal surfaces, producing serum, which made me keep them in longer than I should otherwise have done. On the evening of the second day after operation, during a bad attack of coughing, the temperature and pulse both touched their highest limits, $102.8^{\circ}$ and $106^{\circ}$ respectively. There was no perceptible change in the colour of the skin for more than a week, and the urine, after being a little paler on the third day, became again so dark that we feared there was still some obstruction to the escape of bile. This fear was banished by a copions stool of good colour on the fifth day; and after this the urine rauidly regained its normal appearance.

The wound did not heal very kindly, being much interfered with by her violent coughing fits. The stoois passed contained considerable quantities of fine bile sand for some time after the operation, and it was fully three weeks before the skin began to regain a natural colour. She went home into Shropshire on March 22nd, just six weeks after the operation. It is interesting to note that her mother had jaundice at 45, and again at 55, passing gall stones after the latter attack. In November, 1886, at the time of her visit to Torquay, the patient weighed $146 \mathrm{lbs}$., and emaciation was rapid after this up to the time of operation. She now weighs $162 \mathrm{lbs}$.

CASE IF.-E. S., aged 56, married thirty-five years, and mother of twelve children, the youngest being 13 . The present illness began with pains over the gall bladder in May, 1886; after several attacks of pain, jaundice came on, and when I first saw her she was very deeply jaundiced. She stated that she had had some similar attacks of pain two or three years back, but they passed off without further trouble. Severe attacks of pain recur every two or three days, and as they pass off the urine becornes very dark and thick. About every three weeks she has a severe attack of vomiting, bringing up large quantities of bile. Emaciation is marked, and has been rapid of late, and the itching of the skin is very troublesome, so that she scratches herself into sores all over.

On examining the abdomen, I found the liver large, but not nearly so large as in the previous case. I could not map out the gall bladder, and was not nearly so confident that I could feel a stone ; but I did once or twice think I could feel something hard deep down, and pressure here caused great pain, as in the previous case. The vomiting of bile, the varying colour of the urine and stools in this case showed that the obstruction was not complete; but the jaundice was persistent, and the general health was completely breaking down, and, with the fear of ulceration, I had no hesitation in advising operation, though the age and condition of the patient made me decidedly anxious as to the result, for I felt certain that I should have the same difficulty with the contracted gall bladder that I had met with in the other case.

She was admitted into the Samaritan Hospital, and in the presence of Sir Spencer Wells, her own medical attendant, Dr. Henley, of Woodbridge, and many others, I operated on May 13th, Mr. Malcclm assisting, and Mr. Murray giving chloroform. I need not describe the operation in detail ; it was very similar to the other, matting of intestines and omentum being, however, more extensive and difficult to separate. When I could get at the gall bladder I at once felt a large stone in the cystic duct; after openivg the gall bladder this was removed whole without much difficulty, but I found the duct much contracted behind it, and had great difficulty in breaking up and removing two other large stones from the common duct; the deepest of these still had a facet on its lower surface, but I could find no stone either by finger or probe from above, or by palpation outside in the peritoneum, and the after progress of the case makes it probable that this other stone must have found its way into the bowel at some period before the operation.

The large stone first removed weighed 150 grains, the fragments of the two others 210 grains.

The gall bladder was well washed out with solution of corrosive sublimate, and its edges sutured into the wound in the abdominal parietes, a small, red rubber drainage tube being introduced deeply into the common duct, and kept in position by a safety pin.

The very large quantities of bile and bile-stained mucus which were discharged in this case immediately and for a long time after operation, show clearly that the strain on the sutures, and the danger of escape of bile into the peritoneum, must be great in such a case if the bladder is closed and dropped, and I think it should be a rule to provide free drainage of the peritoneum till all danger of such escape has passed away.

There was a sharp rise of temperature and pulse (102 and 116) on the evening of the first day after operation, apparently due to some lung complication. The breathing being shallow and difficult, with severe pain round the right side of the chest, this was relieved by poultices and ice to the head.

The discharge of bile and bilious fluid from the gall bladder was very free and very offensive ; it continued very free as long as the drainage tube was kept in (sixth day), but when the tube was $r \in$ moved rapidly decreased, apparently finding its way down into the bowel. Trie buwels moved freely on the fifth day afier a single dose of white nixture; the stools were light grey; they were carefully examined, but no stone or fragments of stone could be found. The temperature was normal on the eighth day, and then for some time slightly subnormal, as it had been before tho operation. The patient suffered a great deal more pain in the region of the gall bladder than the other, whose gall bladder was not fastened up into the abdominal incision.

The discharge from the wound practically ceased on June 17th, rather more than a month after the operation, and the stool was of a natural colour on the following day for the first time. She was up on June 20th, and discharged well on the 28th, just abcut six weeks after operation. The time occupied in recovery thus corresponded in the twn cases. I have recently heard that she is now in good health.

During the time the first of these cases was under my care, I saw with Dr. Waterhouse, at the request of Dr. Broadbent, an elderly lady with an impacted stone, and arranged to operate upon her in the course of a few days; shortly after my visit, however, she passed the stone at stool, and happily escaped surgery. I bave little doubt that my efforts to make sure that her symptoms were due to the presence of an impacted stone forced it through into the bowel, for I could distinctly feel a hard substance deep down in the usual situation, and I made a specially careful examination because there was no distension of the gall bladder and no jaundice, and I was anxious if possible to make out whether the stone was in the cystic or common duct. The 
successful result of manipulation in this case might be thought iencouraging for persevering manipulation as a rule of practice in these cases before subjecting the patient to surgery ; but certainly, in the second case at any rate, no good could have resulted from any amount of manipulation, and it must in any case be attended with very serious risk of perforation. An acquaintance of my own died after a few hours' illness from the duct becoming gangrenous from the pressure of a large stone, though he had suffered but little inconvenience from its presence, and was apparently in his usual health on the morning of the day of his deatb.

When we have to deal with a distended gall bladder and a stone impacted in the cystic duct, the operation for its removal is much more easy than those just described. The bladder is carefully surrounded with carbolised sponges, and aspirated; when empty it is incised and opened, then its stretched walls are easily held forward ontside the abdominal incision, and the necessary manipulations for the removal of the stone or stones are carried on in a large cavity, and without risk of any remains of the fluid contents fouling the peritoneum.

It is very different when the walls of the gall bladler are so con. tracted that they only admit of a very small incision, the edges of which cannot be brought outside or even into the lips of the abdominal incision; not only is the risk of fouling the peritoneum greatly in creased, but the exploring finger is tightly grasped by the contracted and thickened walls of the bladder, and there is no space to pass instruments past it for loosening or breaking up and extracting the stone, only those who have had personal experience of the difficulties to be overcome can realise the extreme care necessary to dislodge the stone without damaging or tearing the duct, and of course if there are, $\varepsilon \mathrm{s}$ in Case II, several stones one below another, the tediousness of the proceeding is greatly increased. Even in the relaxed abdomen of an emaciated woman, all one's ingenuity and perseverance is taxed, and the last stone is far beyond the reach of the longest finger, while the broad chest of a strong man, with thick and, perhaps, fat abdominal parietes, adds enormously to the cifficulty both of the operative procedure of extraction and of the suture of the gall bladder into the inoision. The first case I ever operated upon was that of such a man, and I have always believed that the fatal result was due to poisoning by the complete suppression of bile, this being brought about by the great and constant strain upon the gall biadder and ducts after it was at last securely sutured into the parietal wound.

I have never removed the gall bladder, but I am inclined to think that it would be less dangerous to do so in such a case, than either to leave it forcibly dragged into the wound or sutured and dropped into the peritoneum, with the risk of its becoming distended, so that its contents oozed out batween the sutures. If experience shoild show that complete removal of the gall bladder is nearly as safe as opening it and removing the stones, there would be the great advantage of aroiding any possible future trouble from a fresh formation of stones, but in most cases it would bo extremely difficult to close satisfactorily the wound made by its removal.

A case which I reported at the Brighton meeting of the British Medical Association proves morenver that when the gall bladder has disappeared by atrophy, probably due to inflammatory processes, stones may still form in enormous numbers in the smaller ducts in the liver substance. $18 \div$ ?

The comparatively smooth recovery of the two cases here recorded, after such prolonged and difficult operations, are additions to the proofs which have so rapidly accumulated in the last few years as to marvellous toleration of surgical operations by organs contained in, or partly covered by, the peritoneum, provided we can escape the dangers of septic infection.

\section{A CASE OF ECHINOCOCCUS IN THE ORBIT.}

Read in the Section of Ophthalmology at the Annual Meeting of the British Medical Association in Dublin, August, $188 \%$.

BY PROFESSOR VON ZEHENDER, OF ROSTOCK.

I NEVER met with a case of echinococcus in my ophthalmological practice till lately. Since November last year, I happened to meet with two such cases. One of these does not exactly belong to eye diseases; it was a woman aged 64 years with cataract of the right eye. The operation was performed December 6th, 1886, and succeeded perfectly well. Two nights later the patient complained of great pain in the left side, just below the short ribs, in which place one could feel a deeply situated tumour. The urine was loaded with albumen. The patient, who had previously told me she had never before suffered from any serious illness, was transferred to the wards for internal diseases, and died there January 15th, 1887, about five weeks after the cataract operation. At the post-mortem examination a large hydatic cyst was discovered in the spleen. with extensive in. flammation in the neighbourhood, especially in the kidney.

The other case which I beg to be allowed to relate somewhat more extensively was an echinococcus in the crbit.

The patient, a man aged about 38 years, came into my consulting room November 3rd, 1886, with a well-marked protrusion of the right eyeball. He stated that he had remarked this protrusion not earlier than about six weeks before, and thought it was caused by a cold he had canght. The sight was not much impaired; he could read very small print, though with greater difficulty than with the sound eye. I saw the patient no more till January $16 \mathrm{th}, 1887$. During this interval he had consulted several other medical men. In the meanwhile the protrusion had greatly increased. The patient was scarcely able to recognise the largest letters of Jaeger's test types. Ophthalmoscopically the disc anpeared to be choked (what we call Stauungspapille). The direction of the protrnded eye was inwards and downwards, and in the upper and external part of the eyeball the little finger, pushed forward between the margin of the orbit and the eyeball, could distinctly feel a soft, round, deeply-situated tumour. The nature of this tumour was quite unknown; it was impossible to find out any symptoms for a more accurate diagnosis. It was therefore decided to try to remove the tumour without injuring the eyeball.

The first operation was performed on March 24th, 1887. A large incision was made, dividing the outer commissure of the eyelids, and the entrance to the orbit was freely opened by cutting into the conjunctiva along the upper eyelid. Placing into the opening of the wound the first finger of the left hand, ono could easily reach the tumour and catch hold of it and draw it forwards by means of a sharp hook introduced along the finger. The appearance of the tumour was of a bluish-grey colour, and resembled very much the cyst of an echinococcus, but it was impossible to loosen the tumour and to tear it out, because it was too firmly connected with the neighbouring tissues. By the efforts of tearing the hook made a slit in the membrane, and a great quantity of clear fluid escaped, whereupon the cyst retired quite into the depths of the orbit. The diagnosis of echinococcus seemed to be without any doubt, and, if so, needless to con. tinue to extract the collapsed cyst. The eye was bandaged with a weak solution of chloride of mercury, and the patient was brought to bed.

The first result of this apparently not important operation was a considerable swelling of the upper eyelid and the whole right side of the face, extending even to the upper eyelid and the surroundings of the other side. After a few days the swelling began to subside, but the protrusion of the eyeball was not much lessened, and, what had not existed before, a tremendous fold of the conjunctiva projected between the eyeball and the under lid. This state of things not changing in the next following weeks, it was firmly decided to make another operation, and this was performed on May 13th, 1887. The first finger of the left hand introduced into the orbit could not find any cyst, but it found a diffuse consistent substance, which it was tried to cut out with the scissors. I succeeded in taking away a piece of this substance about one inch long and half an inch wide, and some small pieces of the cyst followed this extirpation. The rest of the collapsed cyst, which was esteemed to be about the size of a large walnut, was then found embedded in the substance to be cut away, some more of which was still removed, and then the eye was bandaged in the usual antiseptic way.

The recovery from this second operation was easier and quicker than the first time, though the operation itself was much more serious the scissors and the fingers being introduced deeply into the orbit and almost in the neighbourhood of the optic nerve. The swelling round the eye was by far not so extensive as after the first operation, and also the general health of the patient and his sleep were better than formerly. But there was still an enormous fold in the conjunctiva, bulging forwards between the eyeball and the lower lid, not allowing it to retnrn to its natural place. Therefore it was necessary to make a third and last operation. This was performed on January 6 th, 1887, without the administration of chloroform. The projecting fold of the conjunctiva was divided by a deep incision extending the whole length of the eyelid, and then a large portion of the protruding tumour was cut away, to the effect that the lower lid could be replaced in its natural position and kept there by some suitable stitches. Hereupon the eycball gradually retried within the orbit, and the disfigurement was entirely removed, except that the eye remained in a squinting position; it could not be moved upwards and outwards, 